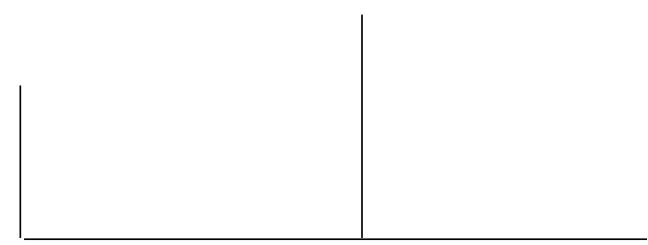

Rev. Latinoam. Psicopat. Fund., VIII, 4, 644-664

\title{
Le thérapeutique et le médical. Du soucie-toi de toi-même au connais-toi toi-même
}

\author{
Roland Gori \\ Marie-José Del Volgo
}

\begin{abstract}
Dans les philosophies antiques et chrétiennes le "souci de soi" (Michel Foucault prescrit que le sujet doit se transformer dans son être pour être capable de vérité et donc apte à la connaissance. Il faut être le thérapeute de soi-même pour accéder véritablement à la connaissance de la vérité. Or notre conception moderne du savoir suppose que toute connaissance impliquant le sujet se révèle non scientifique et se trouve disqualifiée dans sa rationalité et progressivement dans son éthique en tant que guide des conduites humaines. Cette désintrication des régions de la connaissance et de celles du "souci de soi" et du sujet éthique produit un espace vide, un déficit tel que la Science, avec l'aide du Droit, va tenter de combler avec par exemple les modèles réductionnistes, "homme neuronal" ou plus récemment "homme comportemental" allant de pair avec le DSM et les TCC. Rétablir dans ses droits le souci de soi éviterait les dangers de cette passion de l'ordre qui normalise les comportements en les rendant conformes aux normes sociales et éthiques en vigueur.
\end{abstract}

Mots clés: Connaissance, médical, souci de soi, sujet, thérapeutique, vérité 


\section{Le souci de soi}

Michel Foucault a laissé sur le thème du "souci de soi" une œuvre remarquable aux multiples conséquences pour les pratiques thérapeutiques, médicales et non-médicales. Quelles que soient les significations que cette notion de "souci de soi" a pu prendre dans les philosophies antiques et chrétiennes, elles prescrivent toutes que le sujet doit se transformer dans son être pour être capable de vérité et donc apte à la connaissance. Nous sommes ici à l'opposé de notre conception moderne du savoir qui suppose que toute connaissance impliquant le sujet, son ontologie, sa présence dans le savoir, se révèle non scientifique et par conséquent se trouve disqualifiée dans sa rationalité et progressivement dans son éthique en tant que guide des conduites humaines. Dès lors qu'un savoir est scientifiquement exact on peut le transvaser, le déplacer d'un lieu à l'autre, sans requérir pour autant que celui qui le reçoit se révèle apte à l'accueillir. On oublie alors le sens originaire d'informer (Coz, 2003): la forme que l'on donne "prend" dans un matériau préalablement établi et procède ainsi davantage de l' “œuvre” de l'artisan que de la "canalisation” médiatique. Ce point critique où conduit la question de savoir si la connaissance présuppose du sujet une modification de son être, constitue selon la réponse qu'on lui apporte un déterminant essentiel non seulement des malentendus de la médecine mais encore des malaises de notre civilisation. Michel Foucault (2001) nous le rappelle avec le commentaire d'Alcibiade de Platon: la connaissance de soi, le fameux "connais-toi toi-même" de la 


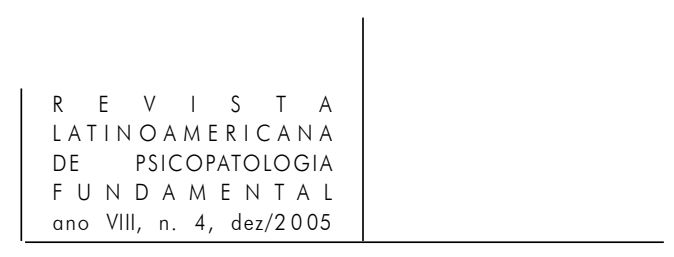

prescription delphique, ${ }^{1}$ demeure dans la culture grecque entièrement subordonnée au "soucie-toi de toi-même". C’est la réponse de Socrate à Alcibiade: "si tu veux connaître le gouvernement des hommes, le fonctionnement de la Cité et de la Nature, commence par te soucier de toi-même, commence par t'occuper de toi". Donc ce n'est pas la connaissance (de soi) qui est prescrite dans cette formule, "connais-toi toi-même", en tant que fondement de la morale, de la spiritualité ou de la politique, c'est le "souci de soi", le souci de "s'occuper de soi-même". Comme le remarque Foucault, lorsque Epicure prescrit également la nécessité de "s'occuper de soi", il emploie le verbe "therapeuein" qui est un verbe à valeurs multiples et dont la polysémie même nous intéresse ici. Il faut donc être le thérapeute de soi-même pour accéder véritablement à la connaissance de la vérité. Or "therapeuein" se réfère à plusieurs actions dont chacun des référentiels tend à absorber dans son "régime" ce "souci de soi":

- Le modèle médical des soins médicaux qui prescrit de devoir s'occuper de l'âme comme du corps.

- Le modèle social ou familial dans le cadre duquel ce verbe renvoie au service qu'un serviteur tend à rendre à son maître.

- Le modèle religieux qui rapporte le "therapeuein" au service des cultes que l'on rend aux divinités.

Cette polysémie du "thérapeutique" innerve l'ensemble des pratiques sociales qui vont s'emparer au cours des siècles et dans la culture occidentale de ce "souci de soi". Et ce jusque par exemple aux malentendus des débats autour de l'encadrement législatif des psychothérapies: ce "souci de soi” dont s'occupe le thérapeute relève-t-il de la médecine? De la psychologie? De la religion "sectaire"? De la pédagogie? De l'individu ou du collectif?

Philon d'Alexandrie distingue, comme de nombreux auteurs Grecs, la thérapeutique et la iatrique. La pratique iatrique s'applique au corps et relève de la médecine alors que la thérapeutique est beaucoup plus large, elle soigne l'âme comme les médecins soignent les corps et elle est vouée à une pratique du culte de l'être.

Rappelons ici que si cette question du "traitement de soi", de son "souci", se pose aujourd'hui à l'occasion du problème de l'encadrement législatif des psychothérapies, cela provient de la logique même de l'exclusion progressive de ce "souci de soi" de la connaissance rationnelle et morale au profit d'un "soi" "objectivé" dans des pratiques sociales d'expertise des comportements jumelées à une science moderne de la nature. Cette exclusion, ce clivage n'existaient pas

1. Une des maximes des Sept Sages inscrite dans le Temple de Delphes. 


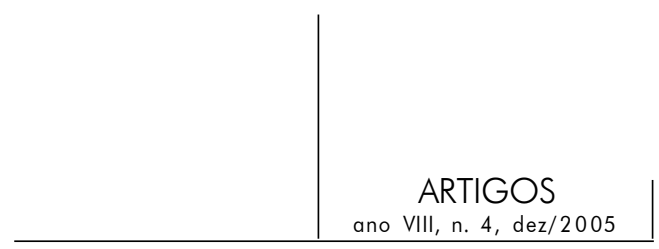

dans la culture antique. Il a constitué sans nul doute le prix à payer pour la connaissance technoscientifique du monde, d'un monde incluant le corps humain, le vivant. C'est ainsi que l'efficacité des connaissances rationnelles des maladies a pu progresser aux dépens de la prise en charge subjective du malade, aux dépens de son souci de soi. Les progrès scientifiques comme les progrès sociaux sont incontestables. Mais cela a un prix et c'est sur ce prix que nous nous attarderons: cette médecine rationnelle et efficace ne serait pas morale si elle ne trouvait pas, d'une manière ou d'une autre, l'occasion d'accueillir le retour du sujet au sein de ses pratiques et de leur théorisation. Peut-être est-ce la raison pour laquelle nous éprouvons un malaise dans notre culture: dans nos pays riches on s'occupe efficacement des maladies par des moyens technoscientifiques sophistiqués mais beaucoup moins des malades et de la valeur ontologique de ce qui leur arrive. Dans les pays pauvres, mais aussi avec les médecines alternatives, le médecin a peu de moyens pour soigner et prévenir les maladies, mais il peut donner beaucoup de son temps et de son attention aux malades. Nous ne pouvons nous résoudre à une telle alternative. Répétons-le encore et encore: nous ne pouvons nous résoudre à choisir entre une médecine technoscientifique redoutablement efficace et une médecine humaine privée de moyens, démunie de reconnaissance scientifique et sociale. La "bio-diversité" des pratiques et des régimes thérapeutiques doit être maintenue non seulement pour la survie de l'espèce mais encore pour des raisons profondément éthiques d'un respect de ces "pluriels singuliers" (Arendt, 1994) que sont les humains. Ce respect conditionne la survie de la civilisation contre les barbaries, que l'intégrisme dont elles relèvent soit religieux, technique ou scientiste.

Reprenons avec Foucault cette notion de "souci de soi". Nous en retrouvons la dimension institutionnelle la plus troublante pour notre époque avec ce groupe de thérapeutes décrit par Philon d'Alexandrie. Cette communauté de "thérapeutes" - doit-on voir là l'archéologie de certains groupes psychothérapeutiques? - aurait été constituée par un groupe de personnes qui se seraient retirées aux environs d'Alexandrie pour "s'occuper de soi" par des pratiques dépliées selon trois axes:

- Un axe cultuel de pratiques religieuses très marquées.

- Un axe intellectuel constitué par des travaux théoriques très poussés.

- Un axe "médical" qui associe aux soins du corps la "guérison" des passions de l'âme, l'étude des rêves et les pratiques d'interprétations allégoriques des Ecritures et des productions psychiques.

Il s'agit d'une "secte" dans la mesure où dans cette "pratique du soi", le retrait de la Cité participe des soins prodigués à l'âme. On ne peut "se soigner" que dans la distinction d'un groupe et d'une communauté qui fait scission du reste du social. L’hôpital s'est aussi fondé sur le retrait social. 


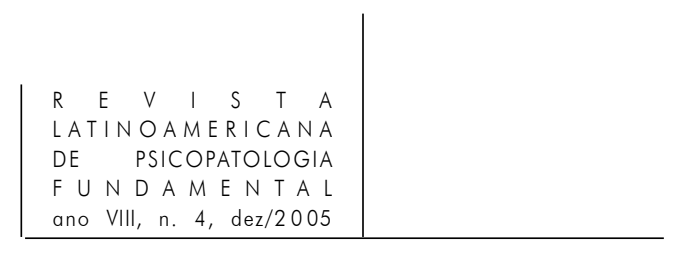

Quelles que soient les formes sociales et culturelles que prennent ce "souci de soi", ce "soin de soi" - y compris le dandysme romantique - on retrouve inexorablement intriqués la santé (du corps), le salut (de l'âme) et l'éthique des conduites vis-à-vis des autres, de soi-même et de Dieu. Cette inclusion réciproque de la santé, du salut et de l'éthique, nous verrons ultérieurement (Gori et Del Volgo, 2005) en quoi les pratiques de santé publique peuvent avoir la prétention de les assumer dans un "hygiénisme” non dénué d’intérêts économiques et politiques: "se sauver et faire son salut" relève d'une manière de se comporter qui va être guidée par les rationalités morale et scientifique. L'EBM, ${ }^{2}$ Evidence Based Medicine, médecine fondée sur les preuves, se trouve élevée à la dignité des Saintes Ecritures auxquelles chaque mortel se réfère dans ses comportements quotidiens et auxquels il sacrifie dans les divers cultes de la "superforme" par des tabous alimentaires et sexuels, comme aux bons vieux temps des religions antiques et des tabous. À moins qu'il ne sacrifie, au nom de la psychosomatique, à un animisme corporel au cours duquel il adhère à la croyance selon laquelle il se fabriquerait lui-même ses maladies. On voit ici la distinction entre "thérapeutique" et "médical”, l'un tendant toujours à se résorber dans l'autre. A notre époque le "iatrique" tant à occuper tout le champ du "thérapeutique". En effet si la culture contemporaine du "soi" se trouve placée sous contrôle sécuritaire, dans une pharmaco-vigilance des comportements et de leurs régimes, alimentaires et sexuels par exemple, rationalisée par la "science" et ses impératifs, on mesure le "chemin" parcouru depuis la vieille formule de L'Alcibiade: "occupetoi de toi-même". Car comme l'écrit Michel Foucault, cette "culture du soi" constitue un "champ de valeurs organisé, avec ses exigences de comportement et son champ technique et théorique associé” (Foucault, 2001, p. 174). Bien sûr, le modèle platonicien qui fait du "souci de soi" une propédeutique au gouvernement des autres en palliant les carences de la "pédagogie" se différencie des modèles plus tardifs (de l'époque hellénistique) qui prônent le "soin de soi" comme "art de vivre", "ascèse" et "conversion à soi-même". Là, on quitte les terres de la pédagogie pour celles de la médecine ou de la théologie. Bien sûr, en prônant l'ascèse et la "renonciation à soi-même" le modèle chrétien diverge radicalement des deux précédents. Mais ce sur quoi nous voudrions insister, après Foucault, consiste à dire que jusqu'au "moment cartésien”, cette connaissance de soi, déduite d'une préoccupation spirituelle et ontologique, n'est absolument pas en position d'exclusion par rapport à la connaissance de la nature. Bien au contraire, elle en est la condition préalable. C'est-à-dire qu'il n'est pas nécessaire

2. Evidence Based Medicine (E.B.M.). L’EBM est un terme créé, dans les années 1980 par un groupe de chercheurs de la Faculté de Médecine Mc Master au Canada, pour désigner une stratégie d'étude clinique. 


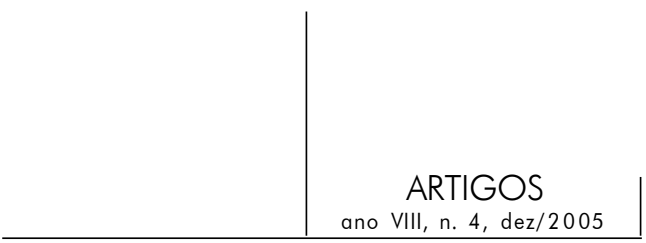

de "naturaliser" le soi pour le connaître objectivement: “'se convertir à soi' est encore une certaine manière de connaître la nature” (ibid.,p. 248).

Dans ces conditions, on conçoit aisément que ce savoir nourri du souci de vérité, de spiritualité et de connaissance de soi, loin de constituer comme à notre époque le négatif de la science, l'autre de la science, en constitue la condition essentielle. A la manière de Khalil Gibran (1991) on pourrait dire: "qu'est-ce que le soleil, si ce n'est un faiseur d'ombres?”. Il n’y a pas à choisir entre la connaissance de soi et la science car l'une demeure sans cesse la condition de l'autre.

\section{Le gouvernement du vivant aujourd'hui}

Nous insistons sur ce point, car sans cela on ne saurait rendre intelligible le mouvement qui s'est accéléré depuis deux siècles et qui vise à promouvoir la science, et en particulier la médecine, en lieu et place de la connaissance de soi. Pour que ce renversement de valeurs soit envisageable, il a fallu ce "tournant destinal” situé par Gadamer (1995) aux origines de la science moderne rationaliste qui a produit une partition, une différenciation des champs du savoir à travers la "régionalisation" des sciences, des arts, de la religion et de la philosophie. Nul doute que sans cette "régionalisation" de la connaissance, la science n'aurait pu atteindre le développement qui a été le sien. Mais nul doute non plus que si la science contemporaine court après sa dimension éthique, c'est bien parce qu'elle lui fait structurellement défaut. Et si l'éthique fait défaut à la science c'est bien parce que celle-ci s'est sans cesse éloignée de la pensée (Heidegger, 1999), de la pensée de ce "souci de soi" qui constituait la finalité de la connaissance antique. Une connaissance qui n'était pas "éthico-poiétique”, pour reprendre le mot de Foucault, n'était pas digne d'intérêt puisqu'elle ne pré-supposait pas la modification de l'être même du sujet pour accéder à la vérité. On voit ici comment la psychanalyse renoue par sa logique avec cette procédure: le sujet n'a accès à la vérité de son être qu'en se modifiant, en retour cet accès à la vérité transforme ses positions subjectives. Et la transformation de sa position subjective lui permet d'avoir un autre accès à la connaissance de son milieu et de la nature dont il fait partie. C'est bien en ce point que la psychanalyse peut contribuer authentiquement à la restitution des dimensions éthiques de la maladie et des soins, sans devoir pour autant renoncer à la science médicale "laïque" qui les ont désacralisés dans leurs techniques. La psychanalyse peut constituer le "passeur" entre ces deux "berges” de la pensée moderne occidentale définitivement éloignées l'une de l'autre: le monde, incluant le sujet objectivé, construit comme objet de la connais- 


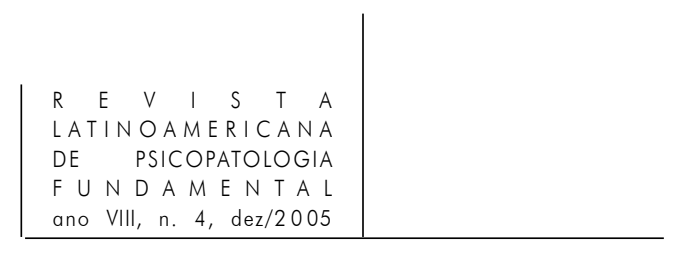

sance rationnelle par la maîtrise de la "tekhnê", et le monde comme "lieu où se manifeste et où s'éprouve le 'soi-même' comme sujet éthique de la vérité” (Foucault, 2001, p. 467). C'est un des objectifs de notre travail: insister sur la portée "éthico-poiétique" de la psychanalyse dans le champ médical où se jouent une connaissance et une modification mutuelles du sujet et du monde dans lequel ce sujet existe. Les questions éthiques actuelles convoquent ce "passeur" faute de pouvoir prendre la mesure de ce qu'elles ont perdu au cours du développement des sciences et des techniques: le sens de l'humain, son site et sa généalogie.

Prenons un exemple parmi d'autre: l'embryon humain est-il humain? (Ouvrage collectif, 1996). Il existe, on le sait, de lourdes ambiguïtés quant aux définitions du "commencement" ou de la "fin" de la vie. Tout dépend de la définition que l'on a et du traitement que l'on fait du mot "vie". Du point de vue physico-chimique le cadavre est toujours biologiquement vivant: "qu'est-ce que la vie?” se demande le physicien Schrödinger(1993) dans un ouvrage qui entremêle physique, biologie et philosophie. Du point de vue juridique, la "vie" peut dépendre d'un autre lorsque la personne juridique n'est plus en mesure de se prononcer sur sa valeur. La "vie" psychique s'avère inséparable des effets de langage et pourtant elle se développe bien avant l'acquisition de la parole. Nous avons donc la plus haute incertitude quant à la définition du "début de la vie" tout comme de sa "fin". Ces définitions procèdent d'un encadrement législatif extrêmement subordonné au cadre culturel d'où il émerge, des pratiques et des techniques qu'il autorise autant qu'elles le déterminent. Il a fallu attendre 1994 pour que les termes "embryon" et "embryon humain" soient expressément utilisés par des textes juridiques (Thouvenin, 1996, p. 27-46). Comme le remarque Dominique Thouvenin, "cela ne signifie nullement que, jusqu'à cette date, le droit ait été indifférent à l'embryon, mais seulement qu'il ne l'appréhendait pas en tant que tel, mais de manière indirecte au travers de la vie humaine” (ibid., p. 27).

Il convient de remarquer, tant avec la loi de 1975 sur l'IVG, qu'avec les lois de Bioéthique de $1994^{3}$ ou les avis du CCNE - Comité Consultatif National d'Éthique (1991) sur les "réductions embryonnaires et fœtales", que l'ensemble de cette technologie de discours procède davantage de la nécessité de réglementer certaines pratiques sociales médicales ou de recherches que d'un "souci" de réflexion éthique et philosophique. Précisons: le statut protecteur de l'embryon a été réclamé afin d'éviter des dérives technico-thérapeutico-commerciales, qui

3. Loi de 1975 sur l'IVG (Interruption Volontaire de Grossesse), elle a autorisé l'IVG en 1975 en France. Lois de Bioéthique de 1994: par une décision du 27 Juillet 1994, le Conseil constitutionnel français proclame le principe constitutionel de sauvegarde de la dignité de la personne humaine. 


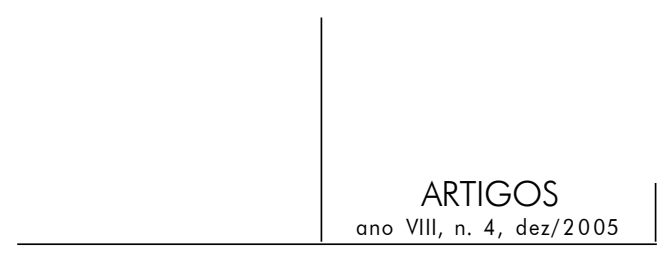

conduiraient à le traiter comme un ensemble de cellules, créé et manipulé à volonté, chose biologique dénuée d’une dignité humaine virtuelle. Car, bien évidemment, le caractère humain prêté à l'embryon s'avère plus important pour celui qui le donne que pour celui qui le reçoit. C'est d'une généalogie de l'humain dans la culture dont il est question. Le souci du législateur a consisté ici encore à encadrer les risques d’instrumentation des éléments et produits du corps humain sans toujours pouvoir parvenir à préciser leur statut ontologique. Et pour cause, puisqu'il s'agissait d'encadrer des pratiques sociales, essentiellement des pratiques médicales et scientifiques, pour lesquelles les débats éthiques et philosophiques, voire politiques n’ont été que les effets. La technique et la science ont ici créé de la philosophie et de l'éthique. Lorsque Jean-François Mattei (1996, p. 47-53), lors du Forum Diderot de 1996, ${ }^{4}$ précise que “l’homme se différencie de l'animal par le caractère sacré qui l'habite” et adhère à la notion de “personne potentielle” pour l'embryon, il le fait à un point d'arrivée critique dans le développement des techniques et des sciences et non à leur point de départ. Les sciences et les techniques se trouvent ainsi rattrapées par le sujet éthique qu'elles avaient forclos.

Toute autre est la démarche des philosophes et des Pères de l'Eglise, par exemple au IIème siècle (Gaspar, 2002), lorsqu'ils s’interrogent sur le statut de l'humain. Leur véritable point de départ est “théologique”. Non seulement parce qu'ils sont théologiens mais bien plutôt parce que la connaissance de l'embryon ne procède pas de son "objectivation" mais constitue une réflexion éthique sur la culture et les pratiques philosophiques dont elle s’inspire. En particulier, le statut de l'embryon conduit à une interrogation critique sur le logos, l'interdit, le sacrifice et le principe d'individualité de l'humain. Ce principe s'avère fort intéressant car il présuppose que l'identité de la personne, sa "totalité" se trouve déjà présente dans chacune de ses parties. Justin, comme Athénagore ou Tertullien, conduisent une réflexion théologique certes hors de notre propos, mais dont l'intérêt consiste à porter leur interrogation religieuse aux limites mêmes des savoirs scientifiques de leur époque afin de répondre à la question: où commence l'humain animé d'une âme? Pour finalement articuler cette question même à une autre énigme: celle du moment où se produit la mort, moment de séparation du corps et de l’âme. Sans devoir nous attarder davantage sur ce débat scolastique, les questions que se posaient les Pères de l'Eglise au IIème siècle, les fantasmes, les passions et les croyances qu'elles mobilisaient autour de l'inceste, du cannibalisme, du commerce du corps humain, des relations au cadavre, des tabous et des interdits qu’elles préconisent, ne sont pas aussi éloignées qu’on pourrait

4. Forum consacré en 1996 au thème "L'embryon humain est-il humain?” et publié aux PUF dans la collection Forum Diderot dirigée par Pierre Fédida et Dominique Lecourt. 


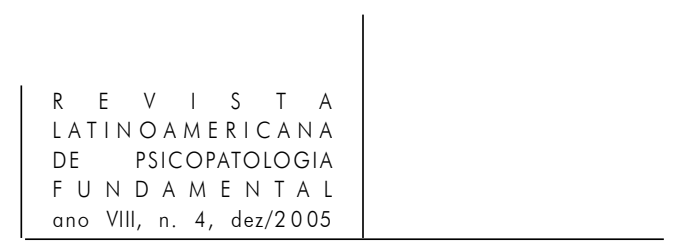

le croire des débats actuels de bioéthique. Seulement, ce qui a radicalement changé depuis le "tournant" historique de la science moderne vient du fait que l'éthique apparaît comme conditionnée par les pratiques sociales de la connaissance et non pas comme les conditionnant.

Précisons encore: la généalogie de l'humain s’impose comme problème éthique à la limite du développement des techniques médicales d'administration du vivant. Cette question fait retour dans les conséquences pratiques d'un mode de connaissance qui en a barré l'accès. Avant le “moment cartésien”, la question éthique était en rapport avec les modalités de la connaissance, encadrant le savoir, voire le précédant.

Prenons un exemple. Marcel Sendrail note que les rabbins, auxquels incombait la charge de pratiquer la circoncision rituelle et d'en surveiller les suites, avaient constaté de loin en loin la fréquence des hémorragies mortelles dans certaines familles. Ils avaient observé la prévalence de ce trouble dans certaines lignées et reconnu son mode de diffusion héréditaire. Cette plus-value de connaissance produite par l'éthique et la pratique religieuse les modifiait en retour en exigeant une réflexion éthique et une exemption rituelle de la circoncision des nouveau-nés dans certaines familles. Il ne s'agit pas bien évidemment de s’abîmer dans la nostalgie des temps obscurs, romantiquement idéalisés, mais plutôt d'évaluer les effets de la régionalisation des savoirs, des modes de subordination du sujet qu'elle prescrit et des pratiques nouvelles qu'elle requiert. De la même manière que le sujet éthique, celui du "souci de soi", tend à son "assujettissement" selon certains modes et à partir d'un type particulier de "substance éthique" selon les époques et les cultures, le sujet "connaissant” quant à lui tend à réaliser sa science selon certains modes et certaines substances historiquement, culturellement et techniquement déterminées. L' "essence de la technique” dont relève notre science moderne, aux dires d'Heidegger (1990), constitue le mode privilégié d'accomplissement de la connaissance. Ce mode "technique" de la connaissance moderne ouvre à l'objectivation du sujet humain dans toutes ses dimensions. Quant à la "substance" éthique de la connaissance, elle se joue principalement dans l'administration du vivant et de son écosystème. C'est-à-dire que cette objectivation du sujet connaissant produit l'oubli même de son être, amnésie de son "souci de soi". Ainsi cette désintrication des régions de la connaissance et de celles du "souci de soi" et du sujet éthique produit un espace vide, un déficit tel que la Science, avec l'aide du Droit, va tenter de le combler à partir des “idéologies” qu'elle produit et des monstres qu'elle fabrique. C'est ce que l'on nomme parfois les "réductionnismes". Pour exemple, mentionnons le réductionnisme génétique qui accrédite l’illusion selon laquelle le livre de la vie d’un individu est déjà "écrit" dans ses gènes. Ou encore le réductionnisme de "l’homme neuronal” de Jean-Pierre Changeux (1983) qui prolonge "l’Homme- 


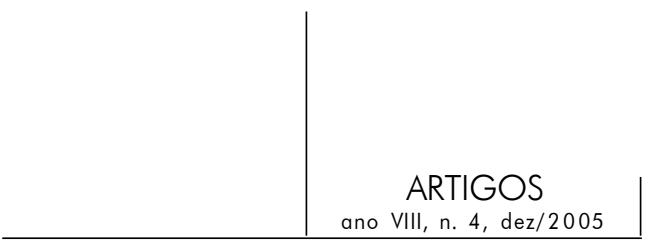

Machine" de La Mettrie (1981) en accréditant la thèse selon laquelle "le cerveau sécrète la pensée comme le foie sécrète la bile”. Les configurations computationnelles des logiciels neuronaux, l'informatique cérébrale, prédétermineraient nos comportements, nos conduites, nos pensées et nos émotions. L'imagerie cérébrale en donnerait les clichés les plus saisissants, saisiearrêt sur l'image de nos émotions et de nos pensées. ${ }^{5}$ Lesquelles pourraient se trouver modulées en fonction de nos goûts et des modes du jour par le pouvoir des psychotropes (Fukuyama, 2002). Et de plus cette manière technique de procéder permet à l'économie des industries pharmaceutiques et des nanotechnologies de tourner. Et nous pourrions toujours au passage "évaluer” nos écarts de conduites et d'humeur par des échelles de comportements dont les scores pourraient nous donner de nous-mêmes, par auto-diagnostic, la marche à suivre pour se "soucier de soi" en "redressant" notre Moi dont les circuits d'apprentissage auraient dysfonctionné.

L'actualité nous apporte un autre exemple des effets rhétoriques de l'annonce de résultats scientifiques locaux et partiels. Le Monde du 22 Mai 2004 et Le Quotidien du Médecin du 26 Mai 2004 n’hésitent pas à renouer avec les vieilles théories des localisations cérébrales des émotions en annonçant la découverte scientifique d' "une zone cérébrale pour les regrets". Il s'agit de travaux expérimentaux de l'Institut des Sciences Cognitives de Lyon (CNRS) ayant comparé l'aptitude aux regrets d'individus normaux et de patients atteints de lésions du cortex orbito-frontal placés en situation expérimentale de décisions à prendre dans des jeux de hasard. L'étude est intéressante dans la mesure où elle montre que le regret renforce le processus d'apprentissage décisionnel chez les sujets normaux. Ce qui ne serait pas le cas chez les sujets orbito-frontaux lésés. Dont acte. Mais de là à écrire que l'équipe française a pu identifier le siège du regret, il y a un pas à franchir qui n'est pas sans rappeler les "slogans publicitaires" et les "rhétoriques de propagande". Cette extension hyperbolique de résultats scientifiques forcément locaux, partiels et expérimentaux repose bien souvent sur une stratégie rhétorique bien connue dans les slogans des campagnes publicitaires.

On le sait là encore avec Michel Foucault, à partir de l'âge classique se développent de nouveaux dispositifs de dressage de l'âme, de redressage des conduites à partir de leurs qualifications morales et psychologiques, notamment grâce à l'expertise psychiatrique. A tel point, écrit Michel Foucault, que par l'expertise "ubuesque" et "grotesque" de la psychiatrie, "le criminel ressemblait

5. Oubliant au passage que “voir le cerveau penser n'est qu’une métaphore poétique” (Edouard Zarifian, Le Monde du 14 Avril 2004). 


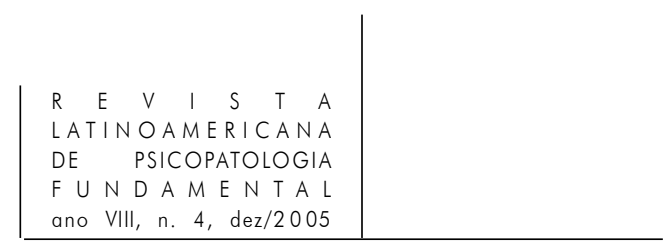

déjà à son crime avant de l'avoir commis". C'est le thème même du film Minority Report. Ce film de Steven Spielberg a pour héros John Anderton, désigné dans le programme "Précrime" de la police comme le meurtrier à venir d'une personne qu'il ne connaît même pas. "Précrime" est une technologie psychique conçue pour arrêter et confondre des meurtriers avant même qu'ils ne commettent leur crime.

Précisons que ce qui se trouve sans cesse amplifié par les techniques actuelles de la psychiatrie comme de la psychologie ou encore de la médecine préventive ou de santé publique, voire de la pédagogie sanitaire - portées par les moyens médiatiques, leur "massification” politique et leur structure de rhétorique publicitaire -, s'organise très tôt comme un quadrillage des conduites par des pouvoirs politiques qui ont su trouver les instruments et les institutions nécessaires à leurs entreprises de normalisation. Nous conviendrons sans peine toutefois que notre tableau se trouve ici dressé à grands traits et que les choses peuvent s'avérer plus compliquées. Dans l'administration contemporaine du vivant, ces dispositifs de gouvernement des conduites, de leur régulation et de leur rééducation procèdent de manière beaucoup plus complexe que ne le laisse pressentir la fresque que nous venons de tracer avec l'aide de Michel Foucault, d'Hannah Arendt, de Gadamer ou de Heidegger. Mais nous souhaitons attirer l'attention du lecteur sur un point historique qui nous paraît essentiel dans l'éclairage des débats actuels autour de la médecine et de la psychanalyse: le "souci de soi" a laissé place à une connaissance rationnelle du monde objectif au sein duquel évolue le sujet vivant et éprouvant. Dès lors, non seulement l'accès à la connaissance ne présuppose pas une modification de l'être du sujet connaissant, mais encore son exclusion constitue la signature même du pedigree authentiquement scientifique. Ce "sujet-là", pour autant qu'il relève d'une "fiction" nécessaire à la cohérence de ses discours et de ses actes il a bien fallu en faire quelque chose. On a parfois laissé le soin à la religion, à l'art ou à la tradition d'en recycler les restes. Mais, c’est à la médecine en général, bien au-delà de la psychiatrie, qu'en a échu les plus beaux et les plus gros morceaux... Les médecins ont sans cesse été requis, depuis plus de cinquante ans, à toujours participer davantage au "gouvernement" du vivant, du prénatal aux fins de vie. Bien au-delà des expertises psychiatriques des comportements, ils ont été convoqués à répondre souvent dans l'urgence et parfois sans formation à cette "dilatation" (Memmi, 2003) des nouvelles demandes sociales. Au point tel que l'un d'entre eux, Thomas Tursz, s'inquiète dans un ouvrage récent (Davant, Tursz, Vallancien, Boncenne, 2003) de cette tendance à considérer que l'assistance et la solidarité sociales relèveraient de la pathologie médicale: "il existe, de nos jours, une forte propension à vouloir rejeter la misère humaine sur le seul système de soins en lui demandant de gérer les mourants, les exclus, les pauvres ou les handicapés” (ibid., p. 180). Tout en 


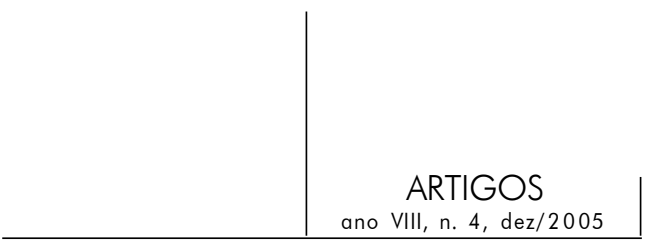

reconnaissant un peu plus loin que “la médecine, en effet, ce n’est pas seulement donner des soins, c’est aussi prendre soin. (...) Il est impossible d'arrêter de prendre soin.” (ibid., p. 186) Et nous revoilà portés aux limites mêmes de la médecine bornée d'un côté par la biologie moléculaire et la génomique et ouverte de l'autre jusqu'à l'horizon des urgences sociales comme à l'infini du gouvernement des conduites. Et ce d'autant plus qu'on assiste à un massif redéploiement des pouvoirs sociaux, professionnels et scientifiques autour du vivant. Redéploiement qui se révèle saturé par des intérêts contradictoires les plus divers, éthiques, politiques, économiques et ontologiques. La médecine ne se contente plus d'exercer un contrôle médical par un acte iatrique, elle étend désormais le pouvoir et les obligations de ses praticiens jusqu'aux limites du "thérapeutique" dans le sens historique de ce terme, soins au corps et à l'âme, services sociaux et pratiques éthiques. Cette "irrésistible dilatation du médical" jusqu’aux confins de la “thérapeutique” (du “souci”) s’est trouvé inévitablement et historiquement marquée par la définition que l'OMS a donné en 1946 de la Santé, alors définie comme "un état de complet bien-être physique, mental et social, qui ne consiste pas seulement en une absence de maladie ou d'infirmité”. L’extension du domaine médical produit un changement de régime épistémologique et éthique considérable dans les pratiques et les idéologies de la santé. Ce changement de régime dans la politique des soins n’a pas d'ailleurs manqué de provoquer des résistances de la part des praticiens eux-mêmes et de leur instance ordinale. Cette irrésistible dilatation du médical a été particulièrement sensible au moment où les médecins se sont trouvés engagés dans de nouvelles pratiques sociales à l'extrême de leur champ de compétence, comme par exemple dans la vérification de la validité des discours biographiques des demandes de contraception, d'IVG ou d'aide médicale à la procréation, ou encore concernant la question de l'euthanasie. Cet accroissement du “magister” médical dans le gouvernement des conduites par la vérification des "conformités biographiques" des demandes (Memmi, 2003) a suscité de nombreux débats dont les lois de bioéthique (1994) comme le code de déontologie médicale de 1995 portent encore la trace.

\section{Malaise dans la santé}

Prenons l'exemple de la situation actuelle en France où nous allons manquer de médecins après avoir manqué d'infirmières. Les Ministres de la Santé qui se succèdent s'activent à trouver des solutions aptes à guérir la France du mal qui la menace et qui pourrait “faire sauter la République”. Cette pénurie de médecins 


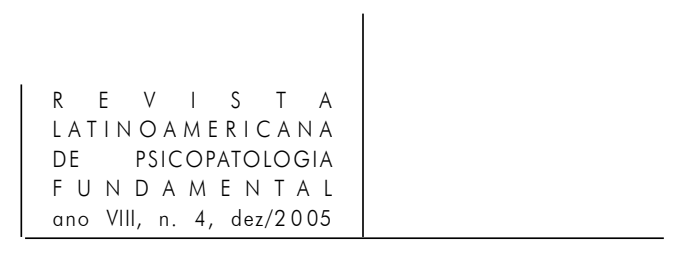

a conduit en 2003 l'ex-Ministre de la Santé, le Pr Jean-François Mattei, à charger de mission le doyen de la Faculté de Médecine, le Pr Yvon Berland, pour qu’il trouve un remède à ce mal. La logique du rapport Berland (2003) suggère un transfert de compétences des médecins vers les auxiliaires médicaux et les paramédicaux pour la réalisation de certains actes qui ne nécessitent pas spécifiquement le savoir-faire du médecin. C'est sans doute une "bonne” solution si on prend la mesure de la dilatation des nouveaux besoins et des nouvelles demandes sociales générés par la prise en charge des populations placées sous protectorat médical, du prénatal à la fin de la vie. Cette constitution d'un "biopouvoir" impliquant les disciplines médicales dans la gestion des populations redistribue les missions traditionnelles des praticiens essentiellement consacrées à la prise en charge des pathologies individuelles. Cette extension du domaine médical amplifie les effets de pénurie du numerus clausus en médecine. L'évidence des faits ne mérite pas qu'on les néglige.

Le développement de la médecine de santé publique, comme celui de l'épidémiologie, ou encore celui de la médecine préventive, ou encore celui de la médecine sociale (scolaire et du travail), s'avère inséparable de cette dilatation du magister médical invité à surveiller et à protéger les populations, la santé morale des groupes (lois anti-sectes) et l'hygiène des individus. On ne saurait comprendre la demande actuelle de devoir inventer de nouveaux dispositifs de prise en charge des souffrances psychiques par de nouveaux professionnels de santé autres que les psychiatres sans rappeler le paysage social, culturel et praxéologique dans lequel cette demande s'inscrit. C'est incontestablement dans ce paysage des professions de santé mentale que surgissent ces "nouveaux venus" du soin que sont les psychothérapeutes de tous horizons, dont a parfois du mal à cerner le relief de leur formation et la légitimité de leur pratique.

En effet, l'extension des besoins et des demandes de santé a paradoxalement produit une "démédicalisation" des prestations et des services médicaux, du moins au sens traditionnel de ces termes. Et ce d'autant plus qu'en devenant technoscientifique, la médecine s'est éloignée de son site clinique au profit des sites technologiques, pharmaceutiques et industriels. Cette dilatation de la pratique médicale étirée entre son bord social et son bord technologique participe de la demande actuelle de redistribution des corps professionnels de santé et participe de cet accroissement de la "nébuleuse" des "psychothérapies".

Nul doute que la psychiatrie en tant que "ventre mou" de la médecine et des sciences du vivant assiste simultanément à l'accroissement des demandes sociales et culturelles à l'adresse de ses praticiens (de la "bébéologie" aux soins palliatifs, en passant par la psychiatrie sociale des conduites délictueuses sans oublier la dépression ordinaire, l'angoisse et la culpabilité générées par la prise en charge des maladies à pronostic péjoratif..., mais tout en conservant 


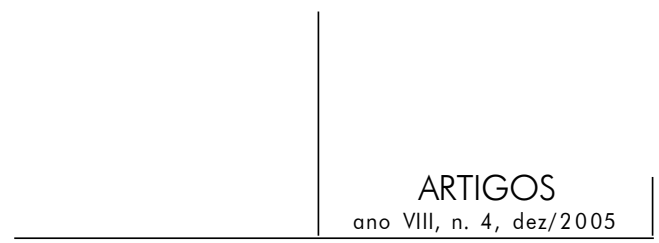

sa mission primordiale de garantir la norme mentale et de gérer la folie) et voit voler en éclats sa consistance épistémologique dès lors que son unité pratique ne cesse de se trouver menacée par son écartèlement entre les sciences du vivant (psychiatrie biologique) et les sciences humaines et sociales (psychothérapies diverses). Dans ce contexte-là, les différentes réformes de l'internat comme celles des modalités de formation des psychiatres ont accru la nécessité d'un transfert des compétences technologiques vers de nouveaux corps professionnels de santé, au premier chef desquels figure à l'évidence celui des psychologues, et plus particulièrement, celui des psychologues “cliniciens”. ${ }^{6}$ Ce transfert s'impose d'autant plus que la promotion idéologique des classifications internationales dans les pratiques et les recherches psychiatriques conduit à favoriser un accouplement entre les nouveaux psychotropes, ce qu'ils représentent socialement (les lobbies pharmaceutiques et industriels), et les recherches dites objectives et scientifiques des comportements et du gouvernement des conduites. Dès lors, la question des psychothérapies et de leur législation perd la valeur d'enjeux corporatistes et critiques qu'elle avait acquise au cours des décennies précédentes. La question désormais est moins de savoir si la psychothérapie doit demeurer le monopole des médecins et des psychiatres qui n’y sont pas formés, que de définir dans un champ protéiforme et fortement sensible socialement les critères de formation et de prestation protégeant le public et les usagers d'influences néfastes, peu scrupuleuses, voire intéressées et moralement dangereuses.

Le psychiatre, à l'exception peut-être de certains pédopsychiatres, ayant désormais en majorité plus et mieux à faire qu'à consacrer son temps aux psychothérapies sans pour autant devoir y renoncer, on peut supposer qu’à court et moyen termes, l'acte psychothérapique (le soin psychique) se verra déléguer, au moins en partie, à d'autres professions de santé, soit en intégrant la dimension psychothérapique de l'acte médical des autres médecins (généralistes, dermatologues, oncologues, algologues etc.), soit, et c’est le plus probable, en le

6. Précisons sur la base du rapport d'Anne Golse (MIRE, 2002) que "par psychologues, nous entendons parler ici des psychologues cliniciens ou spécialisés en psychopathologie et non l'ensemble des psychologues et de la psychologie dont la fameuse 'unité' promue en 1949 par D. Lagache ne semble avoir été qu'une utopie” (p. 29 du rapport Transformation de la psychiatrie et pratiques des psychologues). Cette remarque n’exclue ni la possibilité de constituer d'autres types de formations spécialisées de psychologues adossées à des équipes de recherche pointues mais différentes, ni l'existence d'interfaces permettant le dialogue entre ces psychologies (comme la question de l'éthique et de l'épistémologie, soit principalement des objets méta-théoriques). 


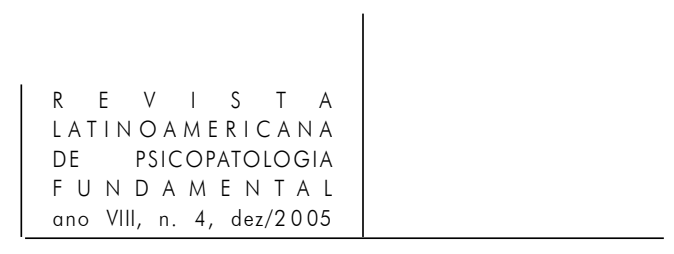

confiant à d'autres soignants au rang desquels figurent au premier chef les psychologues cliniciens.

L'obligation légale de prendre en compte les aspects psychologiques du patient à l'hôpital est récente en France, elle date de 1991. Cette loi - n. 91.748 du 31 juillet 1991 - portant réforme hospitalière oblige l'institution hospitalière dans son ensemble à prendre en compte les aspects psychologiques du patient: "les établissements de santé publics et privés, assurent les examens de diagnostic, la surveillance et le traitement des malades, des blessés et des femmes enceintes en tenant compte des aspects psychologiques du patient". Dans le contexte de cette nouvelle mission hospitalière, le psychologue clinicien occupe une place originale par la prise en considération de la souffrance du malade, mais aussi de sa famille et des soignants. Dans cette écoute de "la maladie du malade" (Canguilhem), le psychologue assure de facto une fonction psychothérapeutique bien au-delà de la simple humanisation de la médecine scientifique, du "supplément d’âme” que peuvent apporter les soignants, les religieux ou les bénévoles. L'intervention du psychologue est souvent brève, liée à la durée de l'hospitalisation. Les demandes des services médicaux et chirurgicaux vont s'accroître d'autant plus que les protocoles de soin vont exiger des décisions éthiques à conséquences juridico-financières et humaines considérables. A moins de créer des postes de "bio-éthiciens", cette mission incombera aux psychologues. A condition que pour un bon nombre d'entre eux la clinique ne soit pas seulement le terrain sur lequel se trouve prélevées des données (psychologie de la santé et psychologie clinique quantitative) mais le lieu d'un acte de soin. Cela suppose que soient totalement repensées non seulement la formation du psychologue, ses missions, ses fonctions mais aussi son insertion permanente dans des protocoles d'enseignement et de recherches. L'avenir de la prise en charge thérapeutique de la souffrance psychique se déduira de cette capacité ou de cet échec à se maintenir dans un rapport d'excentration par rapport à la logique médicale qu'elle accompagne sans s'y laisser absorber.

Ne nous y trompons pas, cette médicalisation de l'existence dans la moindre de nos conduites, expertisées de plus en plus précocement et de manière de plus en plus sensible par rapport aux normes, construit les objets et les méthodes dont elle a besoin pour accomplir sa logique qui consiste en la réduction du thérapeutique.

Voici un exemple parmi cent. La sexualité avait, jusqu’à une époque récente, échappé en partie à sa médicalisation grâce à la psychiatrie, à la sexologie et à la psychanalyse. Au point de proposer quasi-systématiquement une hystérisation généralisée des symptômes corporels dans ce domaine. Cette "psychologisation" des "troubles" et "déviances” de la sexualité tout en participant à la construction sociale d'un sujet "psychologico-moral” chargé d'auto-réguler ses conduites, 


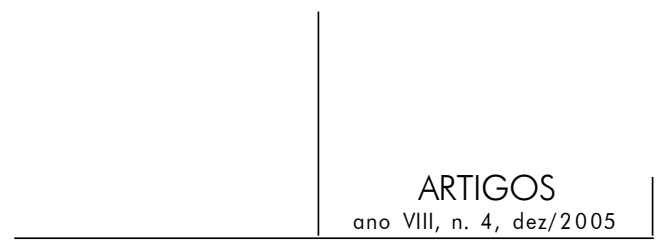

conduisait à reconnaître que quelque chose se dérobait au pouvoir et au savoir du médical. Or depuis une vingtaine d'années, en Amérique d'abord, en Europe ensuite, on assiste au déploiement des stratégies médico-scientifiques (Giami, 2004, p. 77-108) qui rapatrient l'impuissance sexuelle dans le dispositif logique du médical. L'impuissance sexuelle masculine devient une “dysfonction érectile”, un trouble de l'organe pris en charge par les urologues. Les enquêtes épidémiologiques augmentent la fréquence de son diagnostic, les essais cliniques et leurs évaluations conduisent à reconnaître l'efficacité de certains médicaments, comme le Viagra, et favorisent l'instanciation institutionnelle de sociétés savantes, de revues professionnelles et de formations spécialisées (ibid.). Une fois "l'impuissance sexuelle" construite comme un objet médical - et pourquoi pas, puisque ça marche? -, démarche par ailleurs fortement soutenue par les lobbies pharmaceutiques et la demande consumériste des patients, la question de sa légitimité sociale fut posée à partir de celle du remboursement de ces traitements. Sont-ils des aphrodisiaques ou des médicaments? A ce moment là, et bien au-delà des intérêts contradictoires des patients, de l'Etat et des laboratoires pharmaceutiques, ce qui apparaît pour nous, dans ce que Giami appelle une “démédicalisation”, c’est justement un retour du médical aux dépens du thérapeutique. La subordination des normes de santé et de sexualité à l’industrie pharmaceutique ne constitue pas un obstacle à la médicalisation, elle est l'aboutissement de sa logique même: le thérapeutique a disparu au profit d'un gouvernement auto-contrôlé des conduites individuelles, exemptées d'avoir à analyser politiquement ou subjectivement l'implication des sujets qui s'en réclament.

Le transfert de compétences des psychiatres vers les psychologues cliniciens dans la prise en charge de la souffrance psychique a produit une crise au sein même de la psychiatrie. Au point que certains psychiatres ont éprouvé le besoin de confirmer "la nécessité du maintien de leur fonction soignante” et ont refusé la place d'intervenant en deuxième ligne dans la lutte contre le mal-être et la souffrance psychique auxquelles les convoque la logique organisationnelle actuelle. ${ }^{7}$ Là gît le malaise de la psychiatrie actuelle: ou bien le psychiatre renoue avec une pratique psychopathologique référée au paradigme de la psychanalyse pour prendre en charge la souffrance psychique, mais ils ne sont pas assez nombreux et leur formation académique actuelle ne les y prépare pas ou peu ; ou bien ils deviennent des gestionnaires de soins auxquels ils ne sont pas eux-mêmes formés et qu’ils seraient censés encadrer par des expertises scientifiques de

7. Élisabeth Roudinesco a placé cette logique sous l'enseigne des “invasions barbares” in "Les fauxsemblants de l'amendement Accoyer”, Le Monde du 24 novembre 2003. 


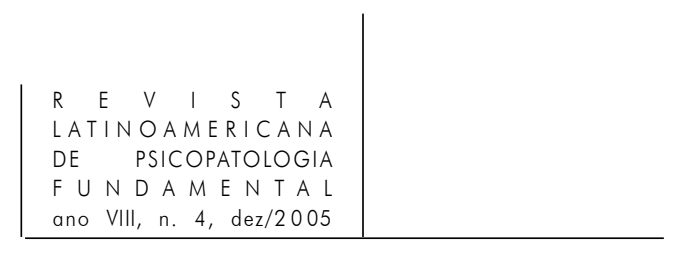

comportement et des prescriptions spécifiques de psychotropes. C'est une des aberrations des rapports Cléry-Melin ${ }^{8}$ et Allilaire et Pichot (2003).

La psychiatrie ayant ainsi "perdu” sa spécificité et l’originalité de son objet (la folie), ses modes particuliers de formations aux pratiques psychopathologiques et aux théories psychodynamiques, étant par ailleurs sollicitée toujours davantage pour une "pharmaco-vigilance" des comportements, risque donc de se médicaliser à outrance pour répondre, au moindre coût, à cette "normalisation" des conduites. Une telle solution médicale des problèmes psychopathologiques rencontrés par la psychiatrie tend à faire de la conduite une maladie et de la souffrance un désordre. Dès lors quand les psychologues refusent de s'inscrire dans la logique des praticiens du soin psychique référée à la psychopathologie clinique et à la psychanalyse, ils tendent à se transformer en auxiliaires médicaux ou en auxiliaires des recherches épidémiologiques. De tels "psychologues en blouse blanche" appelés à la rescousse des psychiatres vont pouvoir, en collaboration avec eux, partager le "grotesque" et l' "ubuesque" des expertises de comportement dénoncées en son temps par Michel Foucault (1999). Psychologues et psychiatres pourraient ainsi s'exonérer d'une "question cynique” posée par Canguilhem: qui les désigne, ces “experts”, comme "instruments de l'instrumentalisme”? "Qu'estce qui pousse ou incline les psychologues à se faire, parmi les hommes, les instruments d'une ambition de traiter l'homme comme un instrument" (Canguilhem, 1956, p. 378).

Pour conclure, disons que nous ne pouvons que nous réjouir du retour du "souci de soi”, de la généalogie du sujet humain et de sa dignité, au sein de la médecine. Sauf que ce retour du "sujet" s'accompagne d'une tentative de le réduire selon la même logique que celle qui a participé originairement à son exclusion en l'objectivant dans ses comportements. Dans cette "passion de l'ordre" qui normalise les comportements en les rendant conformes aux normes sociales et éthiques en vigueur, une haine aussi solide que l'ignorance dont elle se nourrit accomplit son œuvre de mort. Mort du sujet, mort de l'humain, mort de la culture et de la politique, mort de l'histoire et de la durée. Toute une série de "meurtres" s'accomplit pour le plus grand profit de l'ordre, d'un ordre calme et rangé comme un cimetière... Et lorsque les individus eux-mêmes gouverneront leurs conduites et leurs comportements en se corrigeant de leurs écarts par un auto-diagnostic déduit d'un logiciel et traité par des psychotropes, lorsque les solitudes juxtaposées s'entraîneront grâce à des méthodes apprises par les

8. Remis au Ministre de la Santé le 2 octobre 2003, le rapport Cléry-Melin comprend plus de 140 propositions et s'inscrit dans une démarche de santé publique définissant les articulations entre la psychiatrie et la santé mentale. 


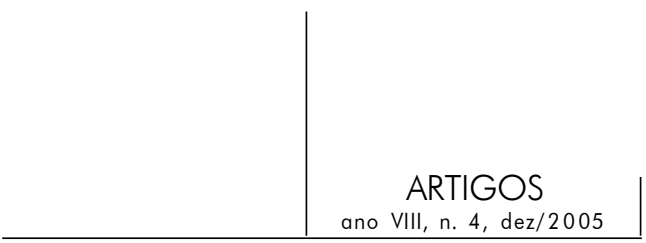

thérapies cognitivo-comportementales, alors l’ordre régnera. La médicalisation des conduites et de la souffrance psychique n'aura plus lieu d'être car l'intériorisation individuelle des normes de santé publique les rendra obsolètes. L'ordre y régnera, mais y aura-t-il encore un sujet pour s'en apercevoir?

Dans cette extrême misère d'une société de travailleurs sans travail, de solitudes amassées, d'emplois du temps perdu sans cesse renouvelés, des matériaux biologiques verront leurs “pièces détachées” échangées automatiquement par des services sociaux et médicaux anonymes et gratuits (le social!), sans cesser pour autant de visionner d'un côté un “jeu vidéo” et de l'autre une émission de "télé-réalité” rediffusée par les Archives du Ministère de la Santé Intérieure, souvenirs des ombres du temps où l'on ne voulait pas souffrir en vain. Jamais l'homme n'aura atteint un tel degré de développement dans sa volonté d’être “distrait” de la Mort et de ses méditations morbides sur l'angoisse et sur le deuil qui lui avaient donné, un temps, l’éphémère illusion d'exister. Ouvert et transparent à toutes les informations qui le traversent sans l'atteindre, un tel homme aura atteint l'état de “stultitia”. Mais ne parlant qu'un nord-américain réduit à la portion congrue de ce qui est techniquement et cognitivement nécessaire, il ne risquera point de rencontrer par inadvertance dans le digest et le zapping des informations pré-sélectionnées dès sa naissance la malédiction de ce terme latin. Michel Foucault nous rappelle que l'état de "stultitia” se déduit de l'ouverture absolue au monde extérieur, d'une transparence totale aux représentations imposées de l'extérieur par la séduction des informations changeantes et éphémères des autres. Le stultus est ouvert aux quatre vents, sans mémoire, sans volonté, dispersé dans le temps et dans l'espace, il laisse la vie s’écouler sans l'avoir vécue. Ce n’est pas une volonté de néant, mais bien plutôt le néant d’une volonté qui a abandonné l'ultime courage d’un Bartleby (Melville, 1995) énonçant mélancoliquement “je préférerais ne pas”. La mélancolie même a été perdue. Ce n'est plus la figure du nomade qui s’impose à une telle culture mais plutôt celle de l'errant, un SDF heureux ouvert au bonheur intemporel assuré par un implant diffusant en permanence et de manière auto-régulée par un logiciel interactif posé dès la naissance les doses optimales de Zoloft et de Ritaline aptes à lui faire oublier la condition tragique de son existence.

Réferénces

Allilaire, Jean-François; Ріснот, Pierre. Rapport au nom d'un groupe de travail. Sur la pratique de la psychothérapie. Bulletin de l'Académie Nationale de Médecine, 187, n. 6, 2003. Disponible en http://www.academie-medecine.fr/actualites/rapports.asp. 


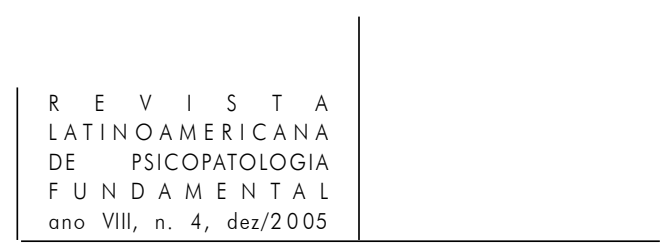

Arendt, Hannah (1958). Condition de l'homme moderne. Paris: Calmann-Lévy, 1994.

Berland, Rapport. Mission "Coopération des professions de santé: le transfert de tâches et de compétences”. 2003. Disponible en http://www.sante.gouv.fr/htm/actu/ berland/sommaire.htm.

CAnguilhem, Georges. Qu'est-ce que la psychologie. In: Etudes d'histoire et de philosophie des sciences. Paris: Vrin, 1956.

Caspar, Philippe. L'embryon au IIème siècle. Paris: L'Harmattan, 2002.

Changeux, Jean-Pierre. L'homme neuronal. Paris: Hachette, 1983.

Coz, Pierre Le. Le moment philosophique de la décision médicale. 2003. 695 pages Thèse (doctorat d'éthique biomédicale), Université d'Aix-Marseille II.

Davant, Jean-Pierre; Tursz, Thomas; Vallancien, Guy avec Boncenne, Pierre. La révolution médicale. Paris: Seuil, 2003.

Foucault, Michel. Les anormaux. Cours au Collège de France, 1974-1975. Paris: Gallimard, 1999.

L’herméneutique du sujet. Cours au Collège de France 1981-1982. Paris: Gallimard, 2001.

FukuYama, Francis. La fin de l'homme. Paris: La Table Ronde, 2002.

GADAMER, Hans Georg. Langage et vérité. Paris: Gallimard, 1995.

GiAmI, Alain. De l'impuissance à la dysfonction érectile destins de la médicalisation de la sexualité. In: Fassin, D. et Memmi, D. (sous la direction). Le government de la vie, mode d'emploi. Paris: Editions de l'EHESS, 2004.

Gibran, Khalil. Le prophète.Paris: Albin Michel, 1991.

Gori, Roland; Del Volgo, Marie-José. La médicalisation de l'existence. Paris: Denoël, 2005.

HeidEgGeR, Martin (1951-1952). Qu'appelle-t-on penser? Paris: PUF, 1999.

Chemins qui ne mènent nulle part. Paris: Gallimard, 1990.

MatTEI, Jean-François. Qu'est-ce que l'embryon? In: L'embryon humain est-il humain? Paris: PUF, 1996. (Forum Diderot).

Melville, Herman (1853). Bartleby. Paris: Le Nouveau Commerce, 1995.

Меммi, Dominique. Faire vivre et laisser mourir. Le gouvernement contemporain de la naissance et de la mort. Paris: Éditions la Découverte, 2003.

MetrRIE, Julien Offroy de La (1748). L'Homme-Machine. Paris: Denoël, 1981.

OuvRage collectif. L'embryon humain est-il humain? Paris: PUF, 1996. (Forum Diderot).

SHRÖDINGER, Erwin (1944). Qu'est-ce que la vie? Paris: Seuil, 1993. 


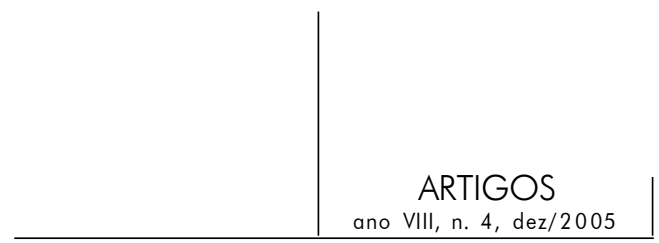

Thouvenin, Dominique. L'embryon humain au regard du droit. In: L'embryon humain est-il humain? Paris: PUF, 1996. (Forum Diderot).

Resumé

Nas filosofias antigas e cristãs o "preocupação consigo mesmo" (Michel Foucault prescreve que o sujeito deve transformar-se no seu ser para ser capaz da verdade e então apto ao conhecimento. Ele deve ser o terapeuta de si mesmo para acessar verdadeiramente ao conhecimento da verdade. Ora, nossa concepção moderna do saber supõe que qualquer conhecimento que implique o sujeito se revela não científica e se encontra desqualificada em sua racionalidade e progressivamente em sua ética enquanto guia das condutas humanas. Esta “desintricação" das regiões do conhecimento e da "preocupação consigo mesmo" e do sujeito ético produz um espaço vazio, um déficit tal que a Ciência, com a ajuda do Direito, vai tentar sanar com por exemplo os modelos reducionistas, "o homem neuronal" ou mais recentemente "homem comportamental", indo de encontro ao DSM e aos TCC. Restabelecer os direitos da "preocupação consigo mesmo" evitaria os perigos desta paixão pela ordem que normatiza os comportamentos tornando-os conformes às leis sociais e éticas em vigor.

Palavras-chave: Conhecimento, médico, “preocupação consigo mesmo”, sujeito, terapêutica, verdade.

En los filósofos antiguos y cristianos el "cuidado de sí” (Michel Foucault) prescribe que el sujeto debe transformarse en su ser para ser capaz de verdad y así estar apto al conocimiento. Es necesario ser el terapeuta de sí mismo para acceder verdaderamente al conocimiento de la verdad. Ahora bien, nuestra concepción moderna del saber supone que todo conocimiento que implique al sujeto se revele no científico y se encuentre descalificado en su racionalidad y progresivamente en su ética en tanto guía de conductas humanas. Separar lo intrincado de las regiones del conocimiento, de aquellas del "cuidado de sí" y del sujeto ético produce un espacio vacío, un déficit tal que la ciencia, con la ayuda del derecho va a tentar llenar con, por ejemplo, modelos reduccionistas; "hombre neuronal" o más recientemente "hombre comportamental" que corren parejo con el DSM y los TCC. Reestablecer en sus derechos el cuidado de sí evitaría los peligros de esta pasión de orden que normatiza los comportamientos tornándolos acordes a las normas sociales y éticas en vigor.

Palabras claves: Conocimiento, medical, cuidado de sí, sujeto, terapéutica, verdad

In classical and Christian philosophies "self-care" (Michel Foucault) states that the subject must undergo transformation in his or her being in order to be capable of truth and thus able to achieve knowledge. You must be your own therapist truly to access 


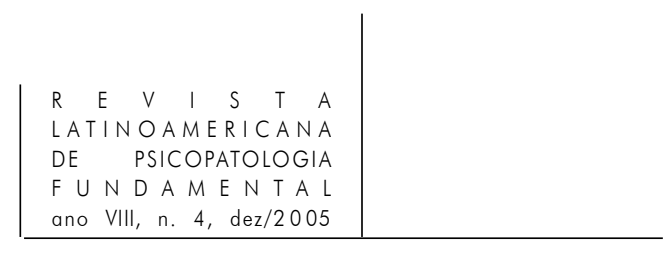

knowledge of truth. Now, our modern conception of knowing supposes that any knowledge involving the subject proves to be non-scientific and is disqualified in its rationality and gradually in its ethics as a guide for human forms of behavior. This diffusion of the regions of knowledge and those of ${ }^{3}$ self-care ${ }^{2}$ and the ethical subject produces an empty space, a deficit that Science, with help from the Law, and attempt to make up for it with, for example, reductionist "neuronal man" or more recently "behavioral man" models that go hand in hand with DSMs and Cognitive Behavioral Therapies. Giving pride of place once more to self-care would avoid the dangers of this passion for order that standardizes forms of behavior by making them comply with prevailing social and ethical standards.

Key words: Knowledge, medical, self-care, subject, therapeutic, truth 